$\frac{1}{6}$

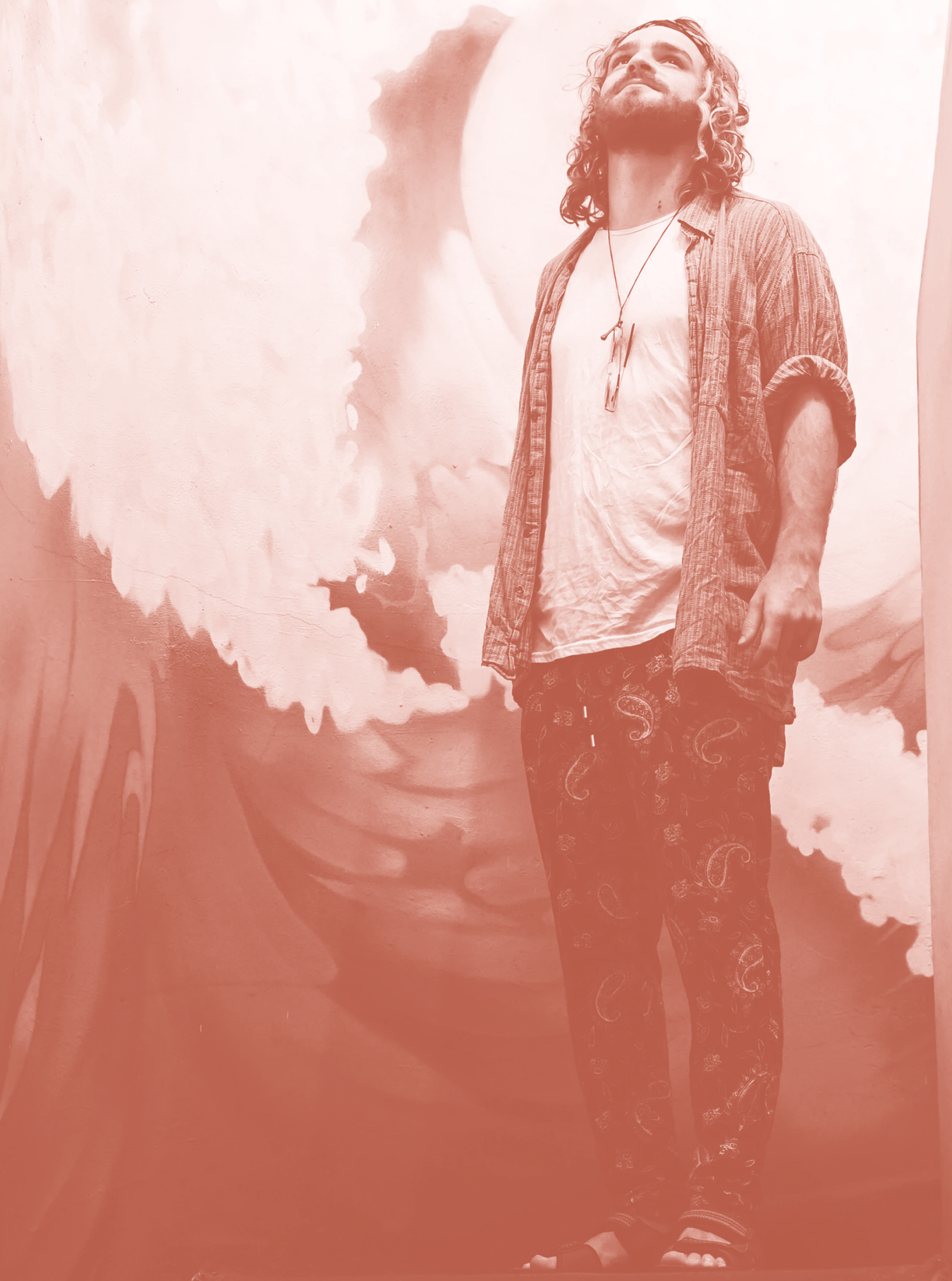




\section{Artistas en espera}

\section{Isabel Cebrián Zarranz, periodista y gestora cultural}

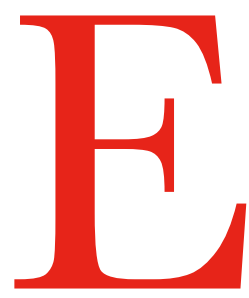

1 llamado Estatuto del artista, aprobado por el Congreso de los Diputados en 2018 tras un proceso de participación transversal y con un amplio consenso político, es un conjunto de setenta y seis medidas recomendadas para proteger los derechos laborales de los artistas profesionales. Una hoja de ruta en la que ya se han dado pasos vía decreto durante el primer gobierno de Pedro Sánchez. Pero la legislatura fallida y el impase electoral han dejado a los trabajadores de la cultura a la espera de la aplicación de unas reformas laborales y fiscales que llevan décadas siendo urgentes.

Por unanimidad: 347 votos, los de todas las formaciones representadas en el Congreso de los Diputados, aprobaron el 6 de septiembre de 2018 el llamado Estatuto del artista. Un informe que recogía el trabajo llevado a cabo durante meses por una subcomisión parlamentaria puesta en marcha a instancias de Unidos Podemos-En Comù Podem-En Marea y que es el primer ladrillo para construir un marco legal, fiscal y laboral para proteger a todos los trabajadores de la cultura y sus necesidades específicas. Un consenso extraño en los tiempos que vivimos.

«Fue muy hermoso vivir el poder contarle a los legisladores cuál es el día a día de la profesión de cómico. Todo el mundo participó en esa comisión con mucha seriedad y pasión», explica la actriz Berta Ojea. Representante de la Unión de Actores y Actrices, para Ojea y su profesión era importante

que se reconociera las particularidades del trabajo de interpretación, es decir, que es un trabajo siempre intermitente, no temporal y que en determinadas situaciones, como la del desempleo, las condiciones no son las mismas que las de otros tipos de trabajo.

Probablemente la misma valoración se puede trasladar al resto de ámbitos de la creación artística, de la escritura a la interpretación musical. Y es por eso por lo que el «feliz acontecimiento» se celebró con júbilo por parte de las organizaciones profesionales que intervinieron en las comparecencias ante esta subcomisión. En total, 31 representantes de facetas de la práctica del arte y la cultura tan dispares como guionistas, actores, escritores, técnicos cinematográficos y 
audiovisuales, ilustradores, artistas audiovisuales, bailarines, músicos, artistas del circo o creadores de videojuegos que representaron en la subcomisión a organizaciones profesionales, asociaciones y sindicatos que ejemplifican la interdisciplinariedad de este proceso.

Lo destaca así Sonia Murcia, directora de la Escuela Superior de Arte Dramático de Murcia, leyendo directamente del texto del Estatuto:

debe resaltarse que los comparecientes fueron convocados de manera consensuada por todos los partidos, evitando que ningún ponente participara de las sesiones llevando una determinada etiqueta política. Para la Subcomisión ha sido esencial apartar las comparecencias del debate partidista. Por otro lado, trabajar desde el consenso ha permitido algo tan importante como racionalizar las aportaciones y asegurar una participación de todos los sectores, que fueron seleccionados siguiendo criterios técnicos y de representación.

Para Marta C. Dehesa, abogada, experta en propiedad intelectual y creatividad gráfica, y compareciente en la subcomisión del Estatuto del artista por la Asociación Profesional de Ilustradores de Madrid (APIM),

fue un acierto no tratar de hacer una ley integral, que tendría mucha complejidad, sino una lista de recomendaciones, medidas y propuestas con 76 puntos que recogen de manera transversal muchos temas que preocupaban a los trabajadores de la cultura, lo que permite que si algunos son problemáticos, se pueden llevar a cabo otros

De ahí los aplausos, felicitaciones y aciertos: por primera vez en la historia, las fuerzas políticas se comprometían con unanimidad a introducir reformas legislativas y a tomar medidas ejecutivas — por ejemplo, campañas dirigidas a los funcionarios de Hacienda y la Seguridad Social para visibilizar el funcionamiento del sector y sus especificidades laborales - que no buscaban subvencionar, sino proteger y favorecer a los creadores teniendo en cuenta algunas particularidades de su trabajo, como la eventualidad, los plazos de la creación, etcétera.

El optimismo del sector que se vio alentado por la velocidad con las que se pusieron en aplicación las primeras medidas. En plena carrera a las elecciones del 28-A, el go- bierno de Pedro Sánchez (PSOE), compuesto tras la moción de censura a Mariano Rajoy (PP) dejaba aprobadas por decreto - y casi in extremis al final de la legislatura - algunas de las medidas más urgentes y para las que había mayor presión social, especialmente aquellas que sobre todo afectaban a los artistas del ámbito público y a los creadores cuyo trabajo está sujeto a derechos de autor.

En el Real Decreto 26/2018 se promulgaban una amalgama de medidas relacionadas con la creación artística y cinematográfica. La decisión con un efecto más directo era la rebaja del IVA en los servicios prestados por artistas, intérpretes, directores y técnicos que ya ha tenido un impacto en aquellos trabajadores que desarrollan su actividad artística para espectáculos públicos. El mismo decreto también recogía una medida relativa a que los artistas en espectáculos públicos puedan cotizar en periodos de inactividad, recogida en ese mismo decreto. A través del Real Decreto 302/2019 se soluciona el problema que hasta ahora obligaba a los artistas mayores a elegir entre la jubilación y el cobro de los derechos de autor, haciendo posible compatibilizar la pensión de jubilación con la práctica de la actividad artística, algo que ya tiene efecto directo en la situación de muchos de nuestros autores y artistas mayores, tanto escritores como actores, músicos... En la trasposición de la normativa europea sobre Propiedad Intelectual (Ley 2/2019) se incluye la posibilidad de que las asociaciones profesionales publiquen listas de tarifas orientativas, una reclamación de sectores creativos como el del diseño y la ilustración.

\section{Del allegro al compás de espera}

Y hasta aquí se puede leer. Un par de días después de que se aprobara la última de estas medidas, España volvía a las urnas y el resultado fragmentario del nuevo parlamento derivaba en una legislatura fallida de 126 días con un gobierno en funciones que no ha avanzado ni un solo paso más en la aplicación de este estatuto. Es mucho lo que queda por hacer, y ni siquiera estas medidas ya aprobadas están teniendo un gran impacto. El optimismo en el sector se ha sustituido, si no por escepticismo, al menos sí por prudencia.

Faltan medidas por aplicar, y las aplicadas no tienen el efecto inmediato que se desearía. Por ejemplo, la aprobación de la compatibilidad de pensiones y trabajo artístico no tiene efecto retroactivo, y deja en tierra de nadie a aquellos creadores que ya han recibido sanciones y multas por simultanear el cobro de pensiones y derechos de autor. Y este paquete de medidas, como recuerda Sonia Murcia, «soluciona 


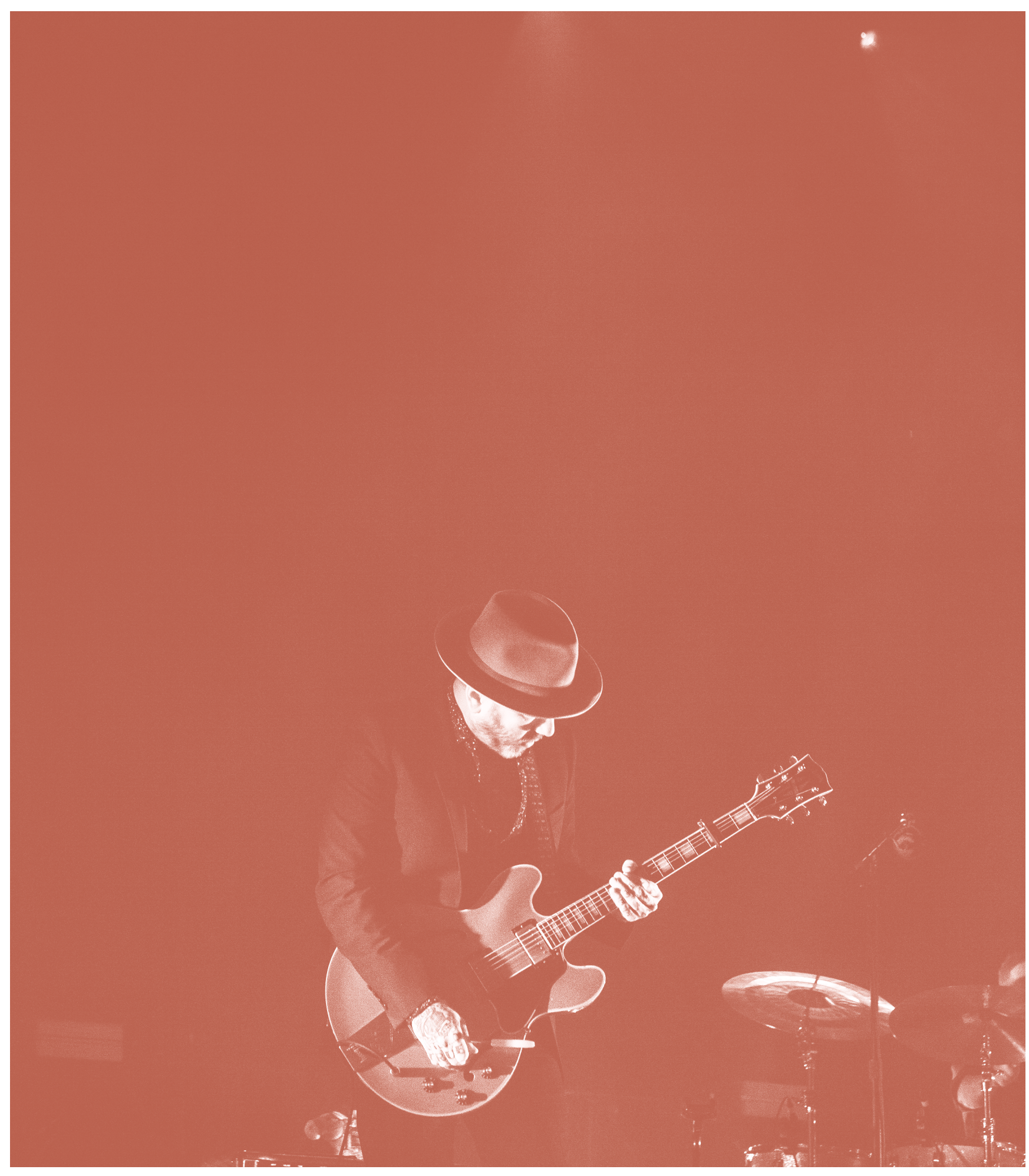


el problema para los creadores pero no para los ejecutantes, es decir, no todos los derechos de propiedad intelectual tienen el mismo tratamiento».

También parece insuficiente para paliar la precariedad del sector la medida que abre la puerta a cotizar en tiempos de inactividad. «Al tener unos ingresos muy bajos ya les cuesta cotizar cuando actúan, mucho más cuando no tengan ningún tipo de ingresos», recuerda Eva Moraga, abogada de la asociación Por\&Para que actúa como asesora para varias asociaciones profesionales de artistas intérpretes, como la Federación Estatal de Compañías y Empresas de Danza.

Las personas consultadas destacan que estas medidas, especialmente aquellas relacionadas con fiscalidad y tributación, afectan a los artistas de espectáculos públicos, pero no a otros que trabajan para otros sectores creativos. Y benefician a quienes trabajan por cuenta ajena, pero no se dice nada sobre los trabajadores que se acogen al RETA, es decir, a los autónomos.

Por suerte, el texto del Estatuto del artista deja el camino pavimentando y hay más de setenta medidas planteadas en el acuerdo y listas para ser puestas en marcha al inicio de una nueva legislatura. Algunas son exigentes y suponen modificar un buen número de leyes, pero otras son de muy fácil aplicación. Por ejemplo, en cuestión de IRPF, el estatuto propone varias posibilidades factibles: aplicar una media de varios años a las cotizaciones y hacer declaraciones de la renta cada tres o cuatro años: un tipo progresivo o una reducción en la base de tributación para tratar de paliar los efectos de las rentas irregulares. Por ejemplo, en el caso de los artistas que trabajan sin remuneración durante uno o dos años en un proyecto como una exposición o un libro y en un solo año reciben todos los ingresos producidos por esa actividad. También se recomienda poder deducir gastos asociados al trabajo, como la formación, los desplazamientos o gastos en hostelería. Y diferenciar los impuestos que se aplican a los ingresos en concepto de anticipo.

Todas estas medidas podrían aplicarse sobre el IRPF, pero el estatuto contempla también la creación de un Régimen Especial de Trabajadores Autónomos para los artistas. Las reformas realizadas no afectan a los trabajadores por cuenta propia y lo que se pide en el Estatuto es garantizar a este gran colectivo - más del 31\% de los trabajadores de la cultura son autónomos - a las prestaciones, ya existentes, por cese de actividad, maternidad, paternidad, lactancia, riesgo de embarazo, enfermedad, accidente de trabajo y compatibilidad de los derechos de autor con la jubilación.
Además, el estatuto ya avanza la posibilidad de hacer las cuotas a la Seguridad Social proporcionales a los ingresos generados y recomienda que la contribución a la misma por el régimen de autónomos sea proporcional a los ingresos generados, además de la gratuidad de cuota gratuita para ingresos por debajo del SMI, rebajar los límites en los que se hace obligatorio cotizar como autónomos a aquellos artistas y artesanos que compatibilicen su actividad con otras por cuenta ajena. En definitiva, un sistema mucho más flexible que evitaría precariedad y fraudes.

Otro tema fundamental es el de la representación colectiva. Explica Marta C. Dehesa:

Especialmente los autónomos creativos, como los ilustradores, somos trabajadores que trabajamos solos en casa de manera individual y necesitamos la fuerza colectiva y la legitimación de las asociaciones profesionales para poder llevar a cabo acciones legales colectivas que nos defiendan de contratos abusivos o cláusulas legales

Simplemente habría que modificar el artículo 11 de la Ley de Enjuiciamiento Civil para dotar a las asociaciones profesionales de legitimación procesal para actuar en nombre de sus asociados frente a abusos y fraudes colectivos en el sector, a semejanza de las asociaciones de consumidores.

Y, sobre todo, las profesionales de la cultura reclaman reconocimiento. En la actualidad, el catálogo de actividades del Impuesto de Actividades Económicas no se adecúa a la realidad de las profesiones artísticas. Un reconocimiento legal de estas profesiones ante Hacienda sería un primer paso para reconocer también sus especificidades individuales y colectivas, pues también se recomienda crear una Sección de Actividades Empresariales para colectivos compuestos por autores.

\section{Lo que queda por hacer}

Setenta y seis medidas de consenso pueden parecer muchas y, sin embargo, algunas medidas que fueron reclamadas por los diferentes colectivos en sus comparecencias no quedaron reflejadas en esta hoja de ruta. Como ejemplo, la promulgación de una Ley Orgánica de Enseñanzas Artísticas Superiores. Recuerda Sonia Murcia: «El estatuto no recoge nada sobre esta demanda necesaria que mantiene una discriminación histórica en el reconocimiento social de 
la formación de la Educación Superior. Ni tampoco en el ámbito de las educación primaria y secundaria» o en materia de igualdad, con medidas que fomenten una mayor diversidad y representación de las mujeres tanto en los diferentes puestos de trabajo del sector cultural, en las representaciones que fomentan las creaciones culturales como películas o series de televisión, como en su presencia en puestos decisivos en las instituciones que rigen la cultura en este país, como reclama Berta Ojea, cuya intervención en la Subcomisión se centró en las medidas por la igualdad.

En definitiva, el Estatuto del artista se celebró como un éxito, pero muchos podrían calificarlo de éxito a medias:

Debo reconocer que la acogida de la normativa de desarrollo del denominado Estatuto del Artista se ha recibido con cierta desconfianza en determinados sectores de la Cultura, tal vez porque cuando empiezan su andadura los reales decreto de desarrollo son siempre complejos.

Sonia Murcia apunta también a «la falta de definición del ámbito subjetivo de la creación artística, más allá de los que se puede interpretar de la Ley de Propiedad Intelectual». El informe señala la dirección a seguir: «se apuesta por que las diferentes personas, actividades y momentos del proceso cultural entren en esta propuesta normativa».

Para Marta G. Dehesa «Se ha quedado en un quiero y no puedo. En nuestra opinión, sigue siendo muy necesario que se apruebe el catálogo profesional, ya que ahora ni en el régimen general ni el RETA se contemplan la realidad de las profesiones creativas». También, que se amplíen las leyes que de momento solo son aplicables a los artistas que trabajan en el ámbito de los espectáculos públicos:

Es un error entender la cultura solo como un espectáculo público, sin tener en cuenta a escritores, ilustradores y fotógrafos, especialmente con las nuevas tecnologías. Sabemos que no es una hoja de ruta per- fecta, pero al menos queremos que esa hoja de ruta se cumpla

\section{Aclara Berta Ojea:}

Se han dado grandes pasos, pero queda un largo recorrido. Es cierto que aunque por parte del gobierno en funciones hemos recibido un gran apoyo, el consenso que ha habido e torno a este tema ha hecho que yo siga dándole a este proceso un voto confianza, porque la puerta no se ha cerrado en ningún momento

Cuando se termina de escribir este reportaje, el parlamento acaba de disolverse y todavía no hay sobre la mesa nuevas propuestas electorales. Sin embargo, el consenso hace pensar que se avanzará en muchas de esas medidas, que supondrían cambios legislativos tremendamente importantes, no solo para los artistas-creadores sino para todo tipo de trabajadores del sector de la cultura, incluyendo a creadores de sectores como el diseño, los videojuegos, gestores culturales o mediadores...

El Estatuto de artista es una hoja de ruta importante, pero como dice Eva Moraga

su efectividad depende ahora de la voluntad política de impulsarlas y llevarlas a cabo. Aunque ha existido el consenso, eso no significa que en el futuro lo siga habiendo. El contexto social, económico y político puede influir en que muchas de ellas se queden en papel mojado.

En resumen: si se quiere, se puede. Cuando se publique este reportaje, acabarán de constituirse unas nuevas Cortes Generales - y, con un poco de suerte, un nuevo Ejecutivo - que tendrán en su mano convertir las recomendaciones en leyes. Normas que hagan posible que se sostenga la actividad profesional de los artistas. La hoja de ruta está escrita y los artistas están a la espera. 